\title{
Phase transition in a stochastic geometry model with applications to statistical mechanics
}

\author{
O. Kazemi ${ }^{1}$, A. Pourdarvish ${ }^{1}$, and J. Sadeghi ${ }^{1}$ \\ ${ }^{1}$ University of Mazandaran
}

December 30, 2021

\begin{abstract}
We study the connected components of the stochastic geometry model on Poisson points which is obtained by connecting points with a probability that depends on their relative position. Equivalently, we investigate the random clusters of the ran- dom connection model defined on the points of a Poisson process in d-dimensional space where the links are added with a particular probability function. We use the thermodynamicrelationsbetweenfreeenergy,entropyandinternalenergytofindthe functions of the cluster size distribution in the statistical mechanics of extensive and non-extensive. By comparing these obtained functions with the probability function predicted by Penrose, we provide a suitable approximate probability function. More- over, we relate this stochastic geometry model to the physics literature by showing how the fluctuations of the thermodynamic quantities of this model correspond to other models when a phase transition (10.1002/mma.6965, 2020) occurs. Also, we obtain the critical point using a new analytical method.
\end{abstract}

\section{Hosted file}

manuscript.pdf available at https://authorea.com/users/453247/articles/551139-phasetransition-in-a-stochastic-geometry-model-with-applications-to-statistical-mechanics 
figures/En/En-eps-converted-to.pdf 
figures/M1/M1-eps-converted-to.pdf 
figures/q16/q16-eps-converted-to.pdf 
figures/qpk1/qpk1-eps-converted-to.pdf 
figures/xv/xv-eps-converted-to.pdf 
figures/cp/cp-eps-converted-to.pdf 